Revista científica, arbitrada e indizada, bajo la modalidad electrónica.

Recibido: $11 / 02 / 2020$

Aceptado: 18/02/2020

\title{
GERENCIA FINANCIERA COMO HERRAMIENTA PARA LA GESTIÓN DE LAS ORGANIZACIONES EMPRESARIALES
}

Financial management as a tool for management of business organizations

Cira Fernández de Pelekais
Florida Global University
cpelekais@ gmail.com
cira.fernandez@ floridaglobal.university
Elmar Aldrin Pelekais
Universidad Autónoma del Caribe
SUMMA University
pelekais@ gmail.com
Carlos Farfán
SUMMA University

\section{RESUMEN}

El objetivo del trabajo es analizar la gerencia financiera como herramienta para la gestión de las organizaciones empresariales. Enmarcándose en el positivismo, paradigma cuantitativo, diseño de campo, transeccional y no experimental de tipo descriptivo. La población cuarenta (40) sujetos. Utilizándose la técnica de la observación mediante encuesta, como instrumento un cuestionario conformado por setenta y un (71) ítems. Los resultados demuestran que el uso de la gestión organizacional en las empresas es realmente efectivo, esto significa que a pesar de existir determinadas debilidades se procuran realizar los procesos efectivamente. Lo referido, precisa que los encargados de liderar los procesos gerenciales, hacen buena aplicación de las estrategias de administración y operaciones.

Palabras clave: Gerencia financiera; Finanzas; Gestión; Organizaciones empresariales.

\begin{abstract}
The objective of the work is to analyze financial management as a tool for the management of business organizations. Framing in positivism, quantitative paradigm, field design, transectional and non-experimental descriptive type. The population forty (40) subjects. Using the observation technique through a survey, as an instrument a questionnaire
\end{abstract}




\section{Revista científica, arbitrada e indizada, bajo la modalidad electrónica.}

consisting of seventy-one (71) items. The results show that the use of organizational management in companies is really effective, this means that despite the existence of certain weaknesses, the processes are effectively carried out. The aforementioned, states that those responsible for leading the management processes, make good application of the administration and operations strategies.

Key words: Financial management; Finance; Management; Business organizations. JEL Classification O16; P34; P43

\section{INTRODUCCIÓN}

Los continuos cambios, la incertidumbre, el caos, la diversidad y pluralidad de opiniones, son elementos que marcan el proceso afrontado por la sociedad, retos que deben enfrentar las organizaciones mediante la búsqueda de herramientas para lograr consenso y alinear los múltiples intereses de los públicos afectos a las mismas, con sus propios intereses.

En este sentido, partiendo de la opinión de Ullman (2014), la gerencia financiera en una empresa pretende plantear diferentes objetivos a cumplir, ya sean estos posibles u óptimos, para que de esta manera, los mismos puedan ser evaluados con posterioridad, en consecuencia, si bien la obtención de estas estrategias son el objetivo final de la misma, esto no se obtiene mediante observaciones vagas de las situaciones financieras de la empresa ya que solo se presenta luego de un extenso y arduo análisis de la totalidad de los efectos, tanto positivos como negativos, que pueden llegar a presentarse para cada decisión que se tome con respecto a la gerencia financiera de la empresa.

Desde este enfoque, según Weston y Copeland (2015):

Es importante que estas decisiones sean tomadas en conjunto y nunca separadamente ya que esta situación podría ser motivo de serios problemas de desacuerdo y convivencia entre los ejecutivos directores de la toma de dichas decisiones, al no tener en cuenta decisiones que pueden llegar a traer consecuencias, que afecten a otros sectores de la empresa. Es por eso que todas las personas encargadas de la gerencia financiera de una empresa, son conscientes de que no existe un plan perfecto. (p.189). 


\section{Revista científica, arbitrada e indizada, bajo la modalidad electrónica.}

Por otro lado, al revisar el estado del arte frente al tema de investigación, es posible encontrar a Cabrera et al., (2017), para quienes el propósito de la gestión financiera en las organizaciones es hacer que se gestionen sus procesos con eficiencia y eficacia, puesto que se trata de propiciar un manejo técnico, humano y transparente tan delicado de la administración y la asignación de recursos financieros. De acuerdo con los citados autores, para cumplir con la demanda del mercado, es necesario un proceso de cambio continuo en materia financiera, por ello la preeminencia de la gestión financiera en las organizaciones, considerando que si una empresa se traza una perspectiva a largo plazo, es imperante un cambio radical en el modelo financiero planteado.

Complementando lo expresado según Gómez et al., (2017), es útil que los gerentes y administradores enfrenten la necesidad de adquirir conocimientos de alto nivel que les permitan tomar decisiones acertadas, para ello se requiere el manejo de instrumentos adecuados desde el punto de vista financiero, a los fines de administrar eficientemente sus organizaciones y conseguir los objetivos instaurados.

De igual forma, Savina y Kuzmina (2015), agregan a lo descrito que el nuevo paradigma de gestión y la necesidad de implementar la estrategia en la empresa, hace que la gerencia de las organizaciones analicen las características, estructura del sistema y la inversión aplicada en los modelos de financiación que utilizan los elementos de la gestión financiera estratégica y operativa. En consecuencia, el análisis de métodos y las herramientas aplicadas, así como la formación de una base conceptual, es el primer paso en el camino hacia la mejora en la eficiencia de la gestión financiera.

Con respecto a lo expuesto, resulta innegable que el principal desafío de cualquier organización, empresarial o no, es gestionar de manera exitosa la administración de sus recursos financieros, porque este aspecto va a garantizar su correcto funcionamiento, además de la sostenibilidad, de allí que implementar la gerencia financiera en los procesos repercute positivamente en el éxito organizacional.

De igual forma, a manera de complementar la función descrita, hace falta tal como lo mencionan Klapper y Luzardi (2019), manejar los conocimientos financieros porque los 


\section{Revista científica, arbitrada e indizada, bajo la modalidad electrónica.}

niveles relativamente bajos de educación financiera exacerban los riesgos del consumidor y del mercado financiero a medida que instrumentos cada vez más complejos ingresan al mercado.

En función de lo planteado, este trabajo esta direccionado en analizar la gerencia financiera como herramienta para la gestión de las organizaciones empresariales. Considerando que es una eficaz herramienta utilizada para apalancar la toma de decisiones, por lo tanto, debe ser abordada de forma integral y metodológicamente clara, a través de un enfoque positivista, tipo cuantitativo, considerando la inclusión de 40 sujetos como población, a los fines de hacer posible los diferentes tipos de análisis en la empresa.

\section{MARCO TEÓRICO}

\subsection{GERENCIA FINANCIERA}

La Gerencia financiera es un aspecto que reviste gran importancia para el funcionamiento y por ende, para la supervivencia de las empresas; su aplicación requiere de personal capacitado o con experiencia en materia financiera en áreas de interés empresarial, pero adaptado a los procesos que se correspondan en la organización.

$\mathrm{Al}$ respecto de lo planteado, Guerra (2015), define la Gerencia financiera como:

La declaración aquello que se pretende hacer en un futuro, y debe tener en cuenta el crecimiento esperado; las interacciones entre financiación e investigación; opciones sobre investigación y financiación y líneas de negocios; la prevención de sorpresas definiendo lo que puede suceder ante diferentes acontecimientos y la factibilidad ante objetivos y metas. (p.355).

En consecuencia, según el citado autor, la Gerencia financiera es una técnica que reúne un conjunto de métodos, instrumentos y objetivos con el fin de establecer en una empresa pronósticos y metas económicas o financieras por alcanzar, tomando en cuenta los medios que se tienen y los que se requieren para lograrlo dentro de un plazo determinado.

Para Weston (2015, p. 254), la Gerencia financiera “implica la elaboración de proyecciones de ventas, ingresos y activos tomando como base estrategias alternativas de 


\section{Revista científica, arbitrada e indizada, bajo la modalidad electrónica.}

producción y mercadotecnia", así como la determinación de los recursos que se necesitan para lograr estas proyecciones. En relación a este aspecto, el objetivo final de esta planificación es un plan financiero en el que se detalla y describe la táctica financiera de la empresa, además se hacen previsiones al futuro basadas en los diferentes estados contables y financieros de la misma. Por ello, el plan lo que pretende es plantear objetivos a cumplir, es decir, los posibles y los óptimos, para ser evaluados con posterioridad.

De igual forma, Farazo (2015), la define como un proceso de análisis de las influencias mutuas entre las alternativas de investigación y de financiación; proyección de las consecuencias futuras de las decisiones presentes, decisión de las alternativas a adoptar y por ultimo comparación del comportamiento posterior con los objetivos establecidos en el plan financiero.

También se puede decir que la Gerencia financiera es un procedimiento en tres fases para decidir qué acciones se deben realizar en lo futuro para lograr los objetivos trazados: planear lo que se quiere hacer, llevar a cabo lo planeado y verificar la eficiencia de cómo se hizo. Por lo tanto, a través de un presupuesto dará a la empresa una coordinación general de funcionamiento. Bajo estos enfoques, tiene la orientación de prever el futuro inmediato con visión estratégica y tomando el largo plazo como fórmula de proyección.

En definitiva, su implementación es para el futuro seguro y cómodo que se desea obtener. Por ello, al planear indica Frank (2014), se abordan en primer lugar, los objetivos, identificando las cosas que la empresa desearía permitirse adquirir, lo que costarían y cuándo necesitaría el recurso económico para pagarlas. Las personas, a diario, buscan administrar bien su dinero, pero pocos elaboran una planificación para encontrar la forma más eficiente de gastarlo o invertirlo, con el fin de obtener una rentabilidad. Por ello, señala Lincoyan (2015, p. 238), que la gerencia financiera "es un arma de gran importancia con que cuentan las organizaciones en los procesos de toma de decisiones.

Asimismo, con la intención de reforzar lo expresado, Rodríguez (2016), citando a Rosillón (2009), aclara que el valor del análisis de la gestión financiera reside en que permite identificar los aspectos económicos y financieros que exponen las condiciones en las cuales opera una empresa con respecto al nivel de liquidez, solvencia, endeudamiento, 


\section{Revista científica, arbitrada e indizada, bajo la modalidad electrónica.}

eficiencia, rendimiento y rentabilidad, facilitando de esta manera la toma de decisiones gerenciales, económicas y financieras en la actividad organizacional cumplida.

De acuerdo a lo planteado, Leyva Ferreiro (2018), afirma que las estrategias financieras constituyen la piedra angular de la planificación financiera. Pueden definirse como el conjunto de metas, objetivos, políticas, que desde una perspectiva financiera, ordenadas y conciliadas de una manera específica, permiten maximizar la eficiencia, el valor, además del cumplimiento de la estrategia general empresarial. Precisamente esta conceptualización se vincula con lo comentado por los autores citados con anterioridad, y que dan claridad a la explicación evidenciada.

Por otro lado, soportando lo señalado anteriormente, Alpízar (2013, p.25), citado por Murillo y Reyes (2018), asegura que la gestión económico-financiera es “el conjunto de diferentes acciones y procesos interrelacionados entre sí que persiguen el objetivo de asegurarle a la organización, de manera eficiente y efectiva, el soporte financiero y material necesario para su funcionamiento y desarrollo, controlando rigurosamente los recursos y empleándolos racionalmente".

\subsection{PROCESOS DE GERENCIA FINANCIERA A CORTO PLAZO}

De acuerdo con Poch (2014), la gerencia financiera es un proceso en virtud del cual se proyectan y fijan las bases de las actividades financieras, con el objeto de minimizar el riesgo y aprovechar las oportunidades. Según Weston y Copeland (2014), constituye una técnica que reúne un conjunto de métodos e instrumentos como objetivos con el fin de establecer en una empresa pronósticos, metas económicas y financieras por alcanzar, tomando en cuenta los medios que se tienen. En este sentido, la planificación a corto plazo hace posible controlar de manera detallada cada una de las actividades de la organización empresarial, de tal manera que son considerados todos aquellos puntos que involucran un riesgo con el propósito de excluirlos a tiempo, y poder de esta manera, asumir las estrategias adecuadas a la naturaleza empresarial. 


\section{Revista científica, arbitrada e indizada, bajo la modalidad electrónica.}

\subsection{FASES DE LA GERENCIA FINANCIERA A LARGO PLAZO}

Para Serna (2015), las fases son los resultados específicos a largo plazo, más de un año, que la empresa busca lograr mediante su misión. En consecuencia, permanecen generalmente estables por el período considerado que abarca la planeación estratégica. Sin embargo, las metas son mucho más específicas en cuanto a tiempo, factibles de cuantificar; considerando que las mismas son blanco a conseguir anualmente, por ello se establecen, cambian o modifican con mayor frecuencia que las fases a desarrollar.

De acuerdo con Amat (2015), la gerencia financiera de la empresa a largo plazo, se encuentra condicionada por las fases generales que con carácter estratégico desee la conseguir la organización. En este sentido, deberá basar su esencia en la consecución a largo plazo; por lo cual debe considerar una serie de aspectos para lograr una verdadera gerencia financiera, entre los cuales señala: Maximizar el valor y la utilidad para los accionistas, además de asumir decisiones financieras.

\subsection{GESTIÓN ORGANIZACIONAL}

Según lo expresa Serna (2015, p. 55), "es el proceso mediante el cual quienes toman decisiones en una organización, obtienen, procesan y analizan información pertinente, interna y externa, con el fin de evaluar la situación presente de la empresa". De igual manera, se puede considerar según expresa Benavides (2015, p. 65), "un conjunto de acciones en el presente que hace una institución con el objeto de lograr el resultados a futuro".

Como complemento de estas afirmaciones, Hernández y Rodríguez (2015, p. 309), indican "trata sobre las decisiones de efectos duraderos e invariables de la administración y dirección de una empresa o institución en una planeación de largo plazo”. Además agrega, David (2015), que implica también integrar la administración, el marketing, las finanzas y la confiabilidad. La producción y las operaciones, las actividades de investigación y desarrollo, así como los sistemas computarizados de información, para lograr el éxito de la organización. 


\section{Revista científica, arbitrada e indizada, bajo la modalidad electrónica.}

Asimismo, es indudable que depende tanto de factores internos como de la disponibilidad de equipos, el estado de los mismos para operar, el capital humano organizado, los factores de compra y venta de dólares, el comportamiento del personal que labora en la empresa. En relación con las implicaciones antes expuestas, se puede deducir que la Gestión organizacional "no es un plan rígido de largo plazo, sino la evolución de una idea principal (visión rectora) a través de un continuo cambio de circunstancias del entorno donde opera la institución”, según expresa Hernández y Rodríguez (2015, p. 309), la estrategia gobierna su futuro, por lo que la mejor forma de adaptarse al futuro es inventándolo y construyéndolo.

Además, es importante recalcar que se deben adaptar los recursos y habilidades de la organización al entorno cambiante, aprovechando sus oportunidades y evaluando los riesgos en función de objetivos y metas. En aras de lograr una buena gestión, se deben formular sistemáticamente una serie de preguntas.

Según indica Serna (2015), el proceso consiste fundamentalmente en responder a las siguientes preguntas:¿Cuál es el negocio?, ¿cuáles son las características del entorno y la competencia?, dónde están las competencias organizacionales, adónde se quiere llegar, cómo llegar, cómo medir que se estén logrando metas y objetivos, cómo se puede observar.

En lo anteriormente expuesto, se define un negocio, la visión de largo plazo y las estrategias para alcanzarla, con base en el análisis de sus fortalezas, debilidades, oportunidades o amenazas.

Al analizar las propuestas teóricas de los autores examinados, se tiene que tanto Serna (2015), como Benavides (2015), concuerdan que en la Gestión Organizacional, se deben tener claro los objetivos, misión y visión de la empresa para establecer una serie de acciones que generaran resultados a futuro.

Por otra parte, Hernández y Rodríguez (2015), establecen que la gestión organizacional como herramienta directiva, sustenta sus direcciones a largo plazo en estrategias o ideas rectoras que orientan acciones o decisiones de los niveles gerenciales. Adicionalmente a lo planteado, David (2015), explica que permite a una organización ser 


\section{Revista científica, arbitrada e indizada, bajo la modalidad electrónica.}

más productiva que reactiva cuando se trata de darle forma a su futuro; consiente iniciar e influir en las actividades (en vez de limitarse a responder a ellas) y, por lo tanto, ejercer control sobre su propio destino.

Entonces, se puede establecer que la gestión organizacional, es considerada como un proceso administrativo, en donde se desarrollan tres etapas principales como lo son la evaluación, formulación e implementación de la estrategia, basándose en objetivos propuestos para lograr los resultados a largo plazo.

Tomando en consideración estos aspectos, se tiene entendido que en toda organización, son los gerentes o directivos a quienes les corresponde el reto de conducir las acciones, tratando de orientar el proceso hacia una gestión exitosa. Esto, mediante el empleo de teorías como la gerencia estratégica, que incluye como eje central los lineamientos donde se desarrollan fases y estrategias. Estas permiten garantizar el adecuado funcionamiento de las actividades administrativas, aunado a una gestión de los recursos de la misma, así como articular la participación de todos los empleados en la búsqueda de la solución de problemas. Cabe destacar también, que todo supervisor necesita desempeñarse dentro de prácticas administrativas que lo conduzcan a gestionar con éxito. Todo ello, centrado en planificaciones adecuadas a los objetivos perseguidos que involucren actividades interrelacionadas con los subordinados y empleados.

Desde este punto de vista, el enfoque o teoría de la gestión organizacional, se presenta como una excelente alternativa para lograr compromisos institucionales con productividad en las acciones ejecutadas. De acuerdo con Chiavenato (2014, p. 147), "es la realizada en el nivel institucional, cumple la tarea primordial de enfrentar la incertidumbre generada por elementos incontrolables mediante las fases de formulación de planes de acción, auditorias del desempeño y el análisis de brecha”.

Igualmente, se requiere de estrategias para enfrentar el horizonte proyectado, que incluyen las estrategias integradoras, intensivas, diversificadas y de cambios basadas en juicios experienciales y no sólo en datos teóricos. 


\section{Revista científica, arbitrada e indizada, bajo la modalidad electrónica.}

Al trasladar estos planteamientos a pequeñas y medianas empresas, es posible trabajar con diseños de estrategias que ubiquen a la misma con experiencias positivas y vinculadas a la realidad del entorno donde se encuentra. Por otro lado, integrar todos los factores en la toma de decisiones, la visión a largo plazo (filosofía de gestión), la de mediano plazo (planes estratégicos funcionales) y la visión a corto plazo (planes operativos), considerando a su vez los planes específicos que no dan lugar a otras interpretaciones y los planes de uso único, diseñados específicamente para hacer frente a las necesidades en una situación inmediata.

El mismo, consiste en una serie de fases secuenciales que proporcionan una nueva energía y dirección a la empresa, donde se consideran los requerimientos de cada planificación para fundamentar las acciones adicionales con el diseño de estrategias integrativas, intensivas, diversificadas y de cambio, según cada necesidad presentada o plan propuesto.

\subsection{PROCESO DE GESTIÓN}

En esta etapa de la gestión se requiere la formulación de estrategias para incluirlas en el desarrollo de la actividad, las cuales permiten identificar y satisfacer los intereses estratégicos de las organizaciones. En este sentido, Chiavenato (2014, p. 621), "refiere que la formulación de estrategias surge como un conjunto de decisiones sobre las relaciones entre la organización y su ambiente".

En cuanto a la formulación de estrategias, el gerente debe tomar en cuenta la misión de la organización, la visión del futuro, el análisis interno entre otras actividades. En la actualidad, la vieja planeación estratégica centralizada puede ser sustituida por la formulación de estrategias orientada hacia un elevado desempeño de la organización, puesto que estas deben innovar, optimizar, agilizar y adaptar enfatizando en la integración selectiva de las funciones y procesos de la organización.

De igual forma, en esta fase se genera el programa de acción que gobernará el desarrollo de la organización, por lo tanto constituye un conjunto de ideas estratégicas orientadas de lo que se desea alcanzar dentro de cada una de las empresas; sin embargo no 


\section{Revista científica, arbitrada e indizada, bajo la modalidad electrónica.}

es un plan rígido y poco flexible, en este caso se trata de estrategias de continua adaptación por lo que está constituido fundamentalmente por políticas más que por normas y procedimientos.

\subsubsection{MISIÓN}

Para Torres (2013), la misión son propósitos concretos a corto o mediano plazo, que la empresa pretende alcanzar cumpliendo con su misión y de acuerdo a sus creencias y valores. De igual manera, el autor antes señalado expone que un objetivo debe formar parte de la construcción de una misión por lo cual ha de poseer tres componentes básicos: la intención que debe ser concreta; la medida que determina un umbral; y el plazo fija un horizonte temporal.

Al respecto, D’ Arellano (2015), establece que la fijación de los objetivos en el desarrollo de la misión empresarial así como la utilización de ellos en el proceso gerencial y la medición de la ejecución, de manera individual y organizacional en su conjunto, implica que los mismos, se fijen conjunta o participativamente por superiores, asimismo subordinados, ya que el desempeño de éstos últimos se aprecia o mide en término del grado de cumplimiento o logro de tales objetivos.

Por otra parte según Rodríguez (2013), el establecimiento de los objetivos es, en efecto, la determinación de un propósito, y cuando se aplica a una organización empresarial, se convierte en el establecimiento de la razón de su existencia. Afirma el autor, que no existe un único o particular objetivo; algunos son de primordial interés para las personas y organizaciones, y no una parte de la organización misma. Otros son de especial utilidad para la organización y les concierne sólo a aquellos que son miembros

\subsubsection{VISIÓN}

La visión es la definición de lo que la institución se visualiza hacia el futuro -tanto a corto, mediano y largo plazo-, esto es, hacia dónde se quiere llegar; recogiendo así las metas y logros que la organización se plantea. En sí, la visión como plantea Chiavenato (2014), es un conjunto de ideas abstractas que proveen un marco de referencia hacia dónde se quiere llegar. 


\section{Revista científica, arbitrada e indizada, bajo la modalidad electrónica.}

De tal manera que, la visión se elabora en el marco de propósitos definidos anteriormente y consiste en una imagen más concreta del futuro que se desea para las unidades de producción, es decir, representación del futuro que se quiere crear a un plazo determinado, descrita en tiempo presente. Debe mostrar a dónde o a qué situación se quiere llegar. La imagen deber ser tangible, dar inmediata dirección en el futuro y ayudar a establecer prioridades.

En este sentido, debe ser coherente con los propósitos y el concepto adoptado y, sobretodo, más específica y concreta. La visión consiste en reflexionar sobre: ¿qué es lo que en realidad se quiere hacer con la Unidad de Producción, lo que realmente se quiere crear o transformar en lugar del estado y desempeño que guardan en la actualidad?. Hacia dónde se quiere llevar a la Unidad de Producción?. ¿Cómo se describiría el estado futuro al cual se tendería y para lo cual se tendría que llevar a cabo una nueva forma de organizarse?.

Siempre que se quiere algo, se especifica la idea en función del futuro, de lo que se necesita tener y lograr, por ello, se habla de la visión, como punto de partida de todo cambio, indicando con éste el plan de acción de la realidad actual, objetiva a una esperada y deseada, situación que se plantea de igual manera, cuando es imprescindible alcanzar el cambio organizacional. Dentro de este marco de referencia, Barroso (2014, p. 95), manifiesta que la visión "es el punto en el futuro donde se quiere llegar, responde a qué final, proporcionando sentido y trascendental, y se vincula directamente con la filosofía de la organización".

Teniendo en cuanta lo destacado, la visión la definen Bounds y Woods (2014, p. 270), como "un sentido comprendido y compartido en común acerca de donde estará la compañía en el futuro y lo que estará haciendo" es decir, para hablar de compromiso, se debe estudiar a qué se comprometen las personas. En el trabajo, las personas deben sentirse adaptadas a la visión de la institución.

Es por ello, que en las instituciones, los miembros deben reunirse para escuchar sus ideas y opiniones acerca de la organización que desean tener, y luego que cada uno las ha aportado, entre todos deben enunciar la visión reflejada con las distintas posiciones, lo cual posibilita que se sientan parte directa de la misma y que luego se comprometan en trabajar 


\section{Revista científica, arbitrada e indizada, bajo la modalidad electrónica.}

para alcanzar lo que quieren, de esta forma, se está generando sentido de pertenencia, de identidad y lealtad.

\subsection{IMPLEMENTACIÓN DE LA GESTIÓN ORGANIZACIONAL}

El proceso de Gestión según David (2015), consta de tres etapas: formulación, implementación y gerencia financiera como herramienta para la gestión organizacional, las cuales en su conjunto le dan la verdadera consistencia a la labor gerencial, puesto que de esta manera al aplicarlas en las pequeñas y medianas empresas propician el mejoramiento de los procesos asumidos.

Por otra parte, Hernández y Rodríguez (2015, p. 320), indican que las etapas del proceso de gestión implican: a) Fijar objetivos, diagnóstico y pronóstico, b) Definición del problema, c) Definir sustento de cambio, d) Ideas estratégicas de modernización y su escenario, e) Formulación de la estrategia y presupuesto, plan rector institucional, f) Ejecución y dirección, g) Administración, dirección y control de la estrategia.

En el mismo orden de ideas, se puede observar que el proceso de gestión es una actividad constituida por múltiples etapas, desarrolladas por diversos autores de manera distinta, pero que pueden ayudar a conocer tanto los elementos internos como externos de una organización, lo cual facilitaría la supervivencia y crecimiento de la empresa.

Bajo estas premisas, se tiene que Serna (2015, p. 57), divide igualmente el proceso en seis etapas, las cuales son: “a) Diagnóstico Estratégico, b) Direccionamiento Estratégico, c) Gerencia financiera, d) Operacionalización Estratégica, e) Implementación Estratégica y f) Evaluación Estratégica”.

Así pues también se puede inferir, al fijar posición con las teorías, que tanto Serna (2015), como Hernández y Rodríguez (2015), establecen que la Gestión Organizacional, se desarrolla en tres niveles: Planeación estratégica corporativa, planeación estratégica funcional o táctica y planeación estratégica operativa. En contraposición, se tiene que Benavides (2015), argumenta que en el referido proceso se define el análisis ambiental (interno-externo) y la filosofía empresarial (propósito, misión, visión y valores). 


\section{Revista científica, arbitrada e indizada, bajo la modalidad electrónica.}

\subsection{EVALUACIÓN DE LA GESTIÓN ORGANIZACIONAL}

Esta es la etapa final de la Gestión según David (2015), los gerentes necesitan saber de inmediato que ciertas estrategias no están funcionando bien y la gerencia financiera como herramienta es el principal medio para obtener esta información. Quiere decir que es una etapa muy importante puesto que sus resultados muestran el desempeño de una estrategia utilizada, es decir, si ha sido efectiva o no para evaluar los procesos dentro de la empresa.

Concuerda Chiavenato (2014), con lo expresado anteriormente, al afirmar que esta es la fase del proceso en la cual "los responsables de una empresa evalúan si las estrategias tal como fueron implantadas están alcanzando los objetivos de la organización”. Cabe considerar, que los gerentes para controlar cuidan los criterios y las mediciones para evaluar los resultados de la misma, así como también comparan los objetivos planteados y los resultados alcanzados.

\subsection{PROCESOS CONTABLES EN LAS EMPRESAS}

Debido a que los procesos contables soportan parte del proceso de la gerencia financiera, se consideró pertinente esbozar algunas ideas al respecto, en este sentido, para Jiménez Aguirre (2015), la información contable en las organizaciones empresariales es una fuente de oportunidades para la gestión administrativa, fiscal, contable, además de financiera; la contabilidad como sistema de información, a partir de los años noventa, ha sido producto no sólo de las exigencias de las organizaciones empresariales en el orbe global, también, de las organizaciones reguladoras que en la urgencia de dar cuenta de estos nuevos requerimientos, ha propiciado la creación de los estándares internacionales de información financiera, que ha permitido normalizar la presentación de estados financieros a nivel mundial. Contribuyendo eficazmente a la gestión organizacional.

Por su parte, Agreda Palacios (2013), sostiene que el proceso de globalización de las economías, así como el uso de las tecnologías de la información han introducido importantes cambios al entorno financiero, la forma de hacer negocios y el intercambio de información financiera, minimizando barreras derivadas de la distancia física. Cada vez más el dinamismo de los mercados, exige mayor y mejor información, por lo tanto no 


\section{Revista científica, arbitrada e indizada, bajo la modalidad electrónica.}

resulta cuestionable afirmar que, la eficiencia del mercado de capital mundial, depende en gran parte de la disponibilidad y oportunidad de información transparente, comparable y relevante, lo cual beneficia el desarrollo y ejecución de los procesos organizacionales.

Complementando lo expuesto Joao et al., (2015), aseguran que en los procesos contables la fase de valuación, es primordial porque en dicha etapa se ponderan las unidades monetarias, los recursos y obligaciones adquiridos por una entidad económica cuando realiza transacciones financieras. Precisamente, según los autores, consiste en la acción de asignar un valor a los recursos y a las obligaciones adquiridas por las empresas para alcanzar los objetivos planteados.

En otro orden Latorre (2016), afirma que en un acercamiento a la contabilidad general, así como a sus fases, con la idea de evidenciar el procedimiento que la caracteriza, hace posible identificar la contabilidad de costos; la cual al ser utilizada en las empresas cumpliendo uno de los principios básicos orientado a la efectividad, garantiza elementos con base a un conjunto de indicadores que apalancan la toma de decisiones administrativas, lo cual incide de manera directa en los procesos contables de cualquier organización.

\subsection{PLANIFICACIÓN FINANCIERA}

Otro aspecto que por su relevancia necesita abordarse es la planificación financiera, la cual según Paredes (2017), constituye uno de los componentes clave para la gestión efectiva de toda organización, su ejecución contribuye con la administración organizacional impulsada para alcanzar los objetivos previa a la operatividad de las funciones relacionadas que soportan las finanzas corporativas, por lo cual resulta imprescindible la definición de sistemas de evaluación y control que garanticen óptimos resultados financiero-operacional en el contexto organizacional que amerite su aplicación. Su alcance permite direccionar la efectiva funcionalidad de procesos administrativos orientados para garantizar la toma de decisiones basadas en el denominado plan financiero concebido en el corto o largo plazo. En este sentido, es operativizada a través de la organización y posterior cumplimiento de acciones.

En función de lo planteado, Pérez y Pérez (2016), enfatizan la relevancia que tiene para el desarrollo eficiente y la rentabilidad empresarial; especificando la necesidad de 


\section{Revista científica, arbitrada e indizada, bajo la modalidad electrónica.}

prever los tributos o impuestos como elementos clave de planificación a partir de la relación establecida entre ellos y las consecuencias desfavorables de no incluirlos. Siendo importante especificar que en la revisión del estado del arte sobre planificación financiera, es evidente una coincidencia por parte de los estudiosos del tema, en relación a la preminencia de este instrumento administrativo en el ciclo de gestión empresarial, considerando que tiene como propósito minimizar el riesgo, aprovechando las oportunidades y los recursos financieros, en los diferentes niveles de toma de decisiones, así como proyección estratégica.

\subsection{ORGANIZACIONES EMPRESARIALES}

En relación a las organizaciones empresariales Arreola et al., (2015), mantienen que para apalancarlas de manera operativa, concurren infinidad de modelos y metodologías articuladas al capital intelectual, desarrolladas tanto teóricamente como en el campo de trabajo, a través del tiempo, lo cual indica que han sido probadas en la práctica organizacional. Por su parte, López et al., (2008), aseguran que los activos intangibles conforman uno de los principales elementos del éxito presente y futuro de las empresas, incrementándose cada vez más las inversiones en los mismos. Con respecto a lo planteado, afirman que está claro un punto vital para cualquier organización y es el referido al hecho que contar con unas instalaciones modernas no garantiza una posición competitiva en los mercados, por el contrario con necesarios procesos de innovación permanente, disponer de un personal con las competencias ajustadas al puesto de trabajo, poseer fidelidad de los clientes, capacidad de liderazgo, relaciones entre trabajadores, credibilidad de los directivos, habilidad para retener y además atraer los mejores profesionales, entre otros aspectos inherentes a las mismas.

\section{METODOLOGÍA}

La investigación que dio origen a este artículo por los elementos que la conforman fue orientada bajo un enfoque positivista, paradigma cuantitativo, descriptiva, con un diseño de campo, transeccional y no experimental. A tal efecto, según Pelekais et al., (2015), este paradigma es objetivo, enfocado en una perspectiva desde afuera, no 


\section{Revista científica, arbitrada e indizada, bajo la modalidad electrónica.}

fundamentada en la realidad, orientada a la comprobación y confirmación de resultados y no en el proceso, se basa en datos sólidos y repetibles, donde se asume una realidad estable. Así mismo, Bavaresco (2010) y Sabino (2014), aseguran que las investigaciones descriptivas van en la búsqueda de aquellos aspectos en los cuales se desea conocer o pretende obtener respuestas, describiendo y analizando sistemáticamente sus características.

Asimismo, Arias (2015), Hernández et al., (2017), señalan que la investigación de campo, consiste en la recolección de datos directamente de los sujetos investigados, o de la realidad donde ocurren los hechos, sin manipular o controlar variable alguna.

En cuanto a la población estuvo constituida por cuarenta (40) sujetos. Para la recolección de la información, fue utilizada la técnica de observación mediante encuesta, siendo elaborado un instrumento tipo cuestionario conformado por setenta y un (71) ítems, el cual fue validado mediante el juicio de cinco (05) expertos, realizándose una prueba piloto a 8 sujetos, a cuyos resultados les fue aplicado el coeficiente Alpha de Cronbach arrojando un índice de confiabilidad de 0,97, lo cual representó un instrumento válido y confiable, posteriormente fue administrado a la población objeto de estudio a los fines de recolectar los datos necesarios para la respectiva tabulación. En el análisis de los datos, se utilizó la estadística descriptiva, indicando las frecuencias en las respuestas y la media aritmética por dimensión e indicadores.

\section{RESULTADOS}

Para iniciar el análisis de la dimensión procesos de gerencia financiera, se desprenden cinco indicadores de relevancia. En este sentido, se observó según la interpretación del baremo previamente diseñado, que la dimensión obtuvo una puntuación de 3,98 lo que se entiende como la existencia de un poco apropiado nivel de gerencia financiera en las organizaciones analizadas. Por lo cual, como lo señala Poch (2014), los procesos de gerencia financiera realmente se cumplen en virtud de lo proyectado, indicando fijamiento de bases de las actividades financieras con el objeto de minimizar el riesgo y aprovechar las oportunidades con los recursos. 


\section{Revista científica, arbitrada e indizada, bajo la modalidad electrónica.}

En consecuencia, para las organizaciones empresariales, constituyen una técnica que reúne un conjunto de métodos, instrumentos como objetivos con el fin de establecer en una empresa pronósticos, metas económicas y financieras por alcanzar, tomando en cuenta los medios que se tienen, como los requerimientos para lograrlo. También, se puede asegurar que la gerencia financiera es un procedimiento en fases para decidir qué acciones se deben realizar en lo futuro.

Los resultados anteriormente señalados, al ser analizados de manera estadística, obtuvieron un puntaje en el baremo de 4,08; lo que evidencia un poco apropiado nivel de gerencia financiera. Coincidiendo con lo señalado por Cárdenas y Nápoles (2015), quienes explican que la administración del efectivo (caja y bancos) es uno de los temas más importantes del presupuesto financiero, o como lo denominan algunos autores, presupuesto de efectivo o tesorería debido a que es uno de los principales problemas a los cuales normalmente se enfrenta un administrador, contralor o gerente.

Por lo tanto, considerando los resultados de cada indicador, el señalado como pronóstico de ingresos, obtuvo un resultado de 4,02; lo cual evidencia según el baremo para medir la gerencia financiera como de poco apropiado nivel. Esto responde a la ejecución de las interrogantes: ¿Se realizan predicciones de las ventas con base en análisis efectuados por un administrador?, ¿Estudia el origen de los fondos financieros que posee la empresa?

En cuanto a "liquidez" y "rentabilidad" se refieren a optimizar resultados, con los cuales los ejecutivos de finanzas no deben estar familiarizados si no por el contrario deben buscar e inventar técnicas que les permitan lograr los mayores beneficios a la administración de recursos disponibles en las organizaciones empresariales analizadas.

Con respecto a las fases de la gerencia financiera, se podrían definir como los puntos previos a los resultados específicos que pretende alcanzar una organización por medio de su misión básica, las fases son esenciales para el éxito de la organización porque establecen un curso, ayudan a la evaluación, producen sinergia, revelan prioridades, información vital para una efectiva toma de decisiones.

Teniendo en cuenta los resultados de la dimensión, esta obtuvo un puntaje de 3,62; 


\section{Revista científica, arbitrada e indizada, bajo la modalidad electrónica.}

lo cual evidencia un poco apropiado nivel de gerencia financiera de las fases de la gerencia financiera en el baremo, esto refleja que las fases se presentan como desafiantes, mensurables, consistentes, razonables y claras. En una empresa, se establecen fases por la compañía entera para cada una de las divisiones. Al respecto David (2015), agrega que las fases anuales son las metas que deben alcanzar las organizaciones a corto plazo, para lograr las fases a largo plazo.

En cuanto a las etapas de aplicación de la gerencia financiera se podrían definir según lo expresado por Thompson (2014), quien asegura no es más que administrar una empresa estratégicamente. Debido a esto se debe desarrollar una visión estratégica de lo que será y hacia dónde va la organización; determinar objetivos, es decir convertir la visión estratégica en resultados; crear una estrategia con el fin de lograr los resultados deseados; poner en práctica y ejecutar la estrategia elegida; finalmente evaluar el desempeño e iniciar ajustes correctivos tanto en la visión como en la dirección a largo plazo.

Asimismo, fueron obtenidos resultados a favor de la gerencia financiera en los aspectos de procesos de gerencia financiera a corto plazo con un puntaje según el baremo de 3,98, para las fases a largo plazo, la cual obtuvo un puntaje de 3,62 y para las etapas de la aplicación de 3,69, lo cual se considera de poco apropiado, a pesar de que se observaron ciertas debilidades en algunos indicadores. Para el análisis del proceso de gestión organizacional, se obtuvo un puntaje de 4,02; que según la interpretación del baremo es alta gestión.

Estos aspectos coinciden con lo expuesto por David (2015), quien explica que la gestión organizacional permite a una empresa ser más productiva que reactiva cuando se trata de darle forma a su futuro; en este sentido, le permite iniciar e influir en las actividades (en vez de limitarse a responder a ellas) y, por lo tanto, ejercer control sobre su propio destino.

Por otro lado, la dimensión implementación de la gestión organizacional, obtuvo un resultado de 4,25 en la media aritmética lo cual se considera según el baremo de medición que se encuentra inserta en la referida variable; por lo tanto se evidencia que el uso de la 


\section{Revista científica, arbitrada e indizada, bajo la modalidad electrónica.}

gestión en las organizaciones empresariales, es realmente efectiva, esto significa que a pesar de existir determinadas debilidades, se procura la ejecución de los procesos efectivamente.

En este mismo orden de ideas, la dimensión evaluación, se entiende como la etapa final, según David (2015), los gerentes necesitan saber de inmediato que ciertas estrategias no están funcionando bien y la gerencia financiera como herramienta es el principal medio para obtener esta información. Quiere decir que es una etapa muy importante puesto que sus resultados muestran el desempeño de una estrategia utilizada, es decir, si ha sido efectiva o no para evaluar los procesos dentro de la empresa.

De igual forma, al analizar la planeación de utilidades, fue obtenido un resultado en el baremo de 3,95; por lo cual se considera según sus niveles de medición como de poco apropiado nivel de gerencia financiera. Siendo importante aclarar que para medir este indicador se realizaron tres preguntas específicas: ¿Al final del ejercicio, las utilidades generadas en el año están en concordancia con las utilidades planificadas?, ¿Se concentra la planificación de utilidades en la elaboración de estados de proformas?

Partiendo que los resultados fueron positivos es decir que existe una alta presencia de los aspectos destacados, se visualiza una coincidencia con lo expresado por Weston (2015), pues esta se refiere a la preparación de estados financieros pro forma, los cuales se consiguen por medio de los estados financieros proforma, donde se muestran los niveles anticipados de ingresos, activos, pasivos y capital social.

Asimismo, el indicador plan de acción obtuvo un resultado de 3,52; el cual se considera de poco apropiado nivel de gerencia financiera. Para la obtención de estos resultados se abordó a la población objeto de estudio a través del siguiente reactivo: ¿Articula estrategias como parte del logro de proyectos empresariales?.

Por último, en cuanto al comportamiento de la variable gerencia financiera, se evidencian resultados a favor de la misma en los aspectos procesos de gerencia financiera a corto plazo con un puntaje según el baremo de 3,98, para las fases de la gerencia financiera a largo plazo se obtuvo un puntaje de 3,62 y para las etapas de la aplicación de la gerencia 


\section{Revista científica, arbitrada e indizada, bajo la modalidad electrónica.}

financiera de 3,69, lo cual se considera de poco apropiado nivel de gerencia financiera, a pesar de que se observaron ciertas debilidades en algunos indicadores.

Estos resultados coinciden con lo expuesto por Weston y Copeland (2014), debido a que la estructura financiera de las organizaciones empresariales analizadas, se encuentra estratificada por lo cual han fijado sus estrategias hacia la búsqueda de nuevos mercados donde la inversión de capitales tenga un régimen de seguridad jurídica sustentable, por esa razón, son tomados en cuenta los posibles obstáculos donde el riesgo financiero tenga un menor impacto; esto permite apalancar procesos como la productividad, calidad y niveles de ingresos.

\section{CONCLUSIONES}

Después de haber cumplido con todas las etapas de la investigación que dio origen a este artículo, el estudio realizado permitió el alcance de los objetivos propuestos, por ello con base a los aspectos teóricos que soportan el mismo y en función de los resultados obtenidos se concluye lo siguiente:

En relación a los procesos de gerencia financiera a corto plazo, donde suele mencionarse la falta de una planificación efectiva como una de las razones por las cuales son generados los problemas financieros y el fracaso, es evidente que la forma de pensar sistemáticamente en el futuro y prever posibles problemas antes de presentarse, es la solución. En consecuencia, es posible afirmar la necesidad de esperar el cumplimiento de un procedimiento lógico y organizado para explorar lo desconocido, siempre apuntalando operativamente la organización.

Con respecto a la premisa de identificar las fases de la gerencia financiera a largo plazo en las organizaciones empresariales, en este proceso de planeación financiera dan por hecho que no es una parte fundamental dentro de los análisis de las empresas o sencillamente lo toman como un indicador poco ilustrativo. Está muy claro que los modelos a veces son complejos, pero realmente necesarios. También se evidencia que no existe una fórmula estándar internacional ni nacional para los modelos financieros, a veces cuenta más 


\section{Revista científica, arbitrada e indizada, bajo la modalidad electrónica.}

un poco de lógica, pero es necesario tener presente que entender el pasado es el primer paso para comprender el futuro de cualquier organización.

Por su parte, con base a las etapas de aplicación de la gerencia financiera en las organizaciones empresariales, resulta incuestionable que la formulación sea uno de los elementos fundamentales de todos los actos realizados por los seres humanos, y máxime, cuando estas actividades pretenden la obtención de objetivos positivos dentro de la operatividad de una empresa.

La enunciación de las etapas de la gerencia financiera, han de definirse sobre la base de las formulaciones operativas de las organizaciones empresariales, así se deben definir estrategias que permitan la ejecución financiera en orden a parámetros lo que repercute en la elaboración del informe final financiero, será básicamente la aplicable dentro de las empresas, se debe basar en técnicas específicas, que pretenden hacer pronósticos de los acontecimientos futuros, pero de igual forma pretende establecer cuáles son las metas y objetivos que se buscan, en el aspecto económico y financiero.

Asimismo, respecto a la implementación de los aspectos tales como; visión, misión, evaluación, tanto interna como externa, deben ser los elementos o factores de la gestión organizacional, que potencien la estrategia en acción, guiando el análisis de elección de estrategias orientadoras de la puesta en marcha de una exitosa empresa.

De la misma forma, se pudo evidenciar que las organizaciones empresariales analizadas, muestran la efectividad de la misma en función de dar respuesta, a través de la implementación de unos procesos coherentes con la correcta aplicación de los principios financieros.

Los resultados permitieron evidenciar que el uso de la gestión organizacional en las empresas es realmente efectivo, esto significa que a pesar de existir determinadas debilidades se procuran realizar los procesos efectivamente.

Lo anteriormente referido, permite definir que en las organizaciones empresariales analizadas, los encargados de liderar los procesos gerenciales, hacen buena aplicación de las estrategias de administración y operaciones que fundamentan la gerencia financiera, 


\section{Revista científica, arbitrada e indizada, bajo la modalidad electrónica.}

siendo que desarrollan un buen proceso de marketing orientando estrategias de finanzas y contabilidad que garantizan su efectividad.

Finalmente, el estudio realizado ha dejado evidente la necesidad de formular líneas de investigación en las cuales puedan adscribirse proyectos relacionados a la gestión financiera en las organizaciones universitarias, abordándolas desde la gerencia de procesos institucionales.

\section{REFERENCIAS BIBLIOGRÁFICAS}

Agreda Palacios, X. (2013). Normas internacionales de información: Avances en su aplicación en Estados Unidos de América, México y Venezuela. Revista del Centro de Investigación. Universidad La Salle, 10 (39), 181-193.

Amat, J (2015). Control de gestión: una perspectiva de dirección. Ediciones Gestión 200 4ta edición. España.

Arias, F. (2015). El proyecto de investigación: introducción a la metodología científica. 5a. ed. Caracas: Editorial Episteme.

Bavaresco, A. (2010). Proceso Metodológico en la Investigación: Cómo hacer un Diseño de Investigación. Editorial EDILUZ Venezuela.

Barroso, B. (2014). Administración y empresa. Mc Graw Hill, Interamericana Editores, S. A., México.

Benavides, C. (2015). Gestión estratégica. Mc Graw Hill, Interamericana Editores, S. A., México.

Bounds, T; Woods, S. (2014). Administración y gerencia en las organizaciones. Mc Graw Hill, Interamericana Editores, S. A., México.

Cabrera, C; Fuentes, M; Cerezo, G. (2017). La gestión financiera aplicada a las organizaciones. Revista Dom. Cien., Vol. 3, núm. 4, octubre, pp. 220-232.

Cárdenas, L; Nápoles, S. (2015). Análisis y pronósticos financieros. Editorial Mc Graw Hill. México.

Chiavenato, I. (2014). Administración de Recursos Humanos. El capital humano de las organizaciones. (Octava edición). Editorial Mc Graw Hill. México.

D’Arellano, V. (2015). Principios administrativos. Pretince-Hall Hispanoamericana S.A., México.

David, F. (2015). La Gerencia Estratégica. Legis Editores S.A., Colombia.

Farazo, S. (2015). Finanzas empresariales y toma de decisiones. Mc Graw Hill. Interamericana Editores. S. A., México.

Frank, T. (2014). Planificación financiera empresarial. Editorial Pearson Addison Weasley. Décima edición, México. 


\section{Revista científica, arbitrada e indizada, bajo la modalidad electrónica.}

Gómez, C; Aristizabal, Ch; Fuentes, D. (2017). Importancia de la Información Financiera para el ejercicio de la Gerencia. Revista Desarrollo Gerencial. Universidad Simón Bolívar-Colombia, 9(2), 88-101.

Guerra, C. (2015). Gerencia financiera en las organizaciones productivas. Mc Graw-Hill. Interamericana Editores, S. A., México.

Hernández, L; Rodríguez, S. (2015). Gestión académica en instituciones universitarias. México: Editorial Mc Graw Hill.

Jiménez Aguirre, R. (2015). Estándares Internacionales de Información Financiera: Algunos efectos en las organizaciones empresariales. Lumina, [S.1.], n. 16, p. 112 135, dic. 2015. ISSN 2619-6174. Disponible en: <http://revistasum.umanizales.edu.co/ojs/index.php/Lumina/article/view/1671/2904 $>$. Fecha de acceso: 09 agost. 2019.

Joao, R; Mateo, V; Da Rosa, C. (2015). Gestión de contabilidad. Un estudio en revistas de Brasil. España y de legua española. Revista de contabilidad y organizaciones, vol. 5 n. 13 (2015) p. 132-150.

Joya Arreola, R; Gámez Adame, L; Ortiz Paniagua, M; Gálvez Fernández, A (2015). Medición del capital intelectual en empresas mexicanas. Retos de la Dirección, 9(1), 79-95.

Klapper, L; Lusardi, A. (2019). Financial literacy and financial resilience: Evidence from around the world. Scientific magazine Financial management. Available in: https://onlinelibrary.wiley.com/doi/pdf/10.1111/fima.12283\#accessDenialLayout

Latorre, F. (2016). Estado del arte de la contabilidad de costos. Revista Publicando, 3(8). 2016, 513-528 ISSN 1390-9304.

Leyva Ferreiro, G. (2018). Indicadores de desempeño empresarial para medir la calidad de las estrategias financieras. Cofin Habana, 12(1), 58-75. Recuperado en 10 de septiembre de 2019, de http://scielo.sld.cu/scielo.php?script=sci_arttext\&pid=S2073$60612018000100005 \& \operatorname{lng}=$ es\&tlng=es.

Lincoyan (2015). Gerencia y análisis financiero. D.F, México. Mc Graw-Hill

López Ruiz, V; Nevado Peña, Domingo; Baños Torres, J. (2008). Indicador sintético de capital intelectual: humano y estructural. Un factor de competitividad. EURE (Santiago), 34(101), 45-70.

Murillo Mora, M; Reyes Espinosa, M. (2018). Gestión financiera con enfoque de procesos en la Universidad Técnica de Manabí: Ecuador. Cofin Habana, 12(1), 32-42. Recuperado en 13 de diciembre de 2019, de http://scielo.sld.cu/scielo.php?script=sci_arttext\&pid=S2073$60612018000100003 \& \operatorname{lng}=$ es\&tlng=es.

Paredes, J. (2017). Planificación financiera ante la perspectiva organizacional en empresas cementeras del estado Zulia-Venezuela. Revista Económicas CUC, 38(1): 105-132. 


\section{Revista científica, arbitrada e indizada, bajo la modalidad electrónica.}

Pelekais, C; El Kadi, O; Seijo, C; Neuman, N. (2015). El ABC de la investigación: Una aproximación teórico-práctica. Ediciones Astro Data. Venezuela.

Pérez, S; Pérez, F. (2016). Planificación financiera de las empresas: El rol de los impuestos o tributos. Revista Publicando, 3 (8): 563-578.

Poch, Y. (2014). Análisis financiero. Editorial Trillas. Barcelona. España

Rodríguez, L. (2013). Administración. Editorial Limusa. D.F, México.

Rodríguez, P. (2016). Gestión financiera en PyMES. Revista Publicando, 3(8). 2016, 588596.

Savina, S; Kuzmina, I. (2015). Improving Financial Management System for Multibusiness Companies. Procedia - Social and Behavioral Sciences 210: 136 - 145.

Serna, G; H., (2015). Gerencia Estratégica. 3R Editores LTDA, Sexta Edición. Colombia.

Thompson, A. (2014). Estrategias y finanzas. Mc Graw-Hill, Interamericana Editores, S. A., México

Torres, C. (2013). Principios administrativos. Mc Graw-Hill, Interamericana Editores, S. A., México.

Ullman, C. (2014). Gerencia financiera. Mc Graw-Hill, Interamericana Editores, S. A., México.

Weston, D. (2015). Gestión financiera en las organizaciones. Prentice Hall Hispanoamericana, México.

Weston, V; Copeland, N. (2015). Finanzas de la empresa. Prentice Hall Hispanoamericana, México. 